\title{
A Novel Random Wireless Packet Multiple Access Method Using CDMA
}

\author{
Christian Schlegel, Roland Kempter, and Preeti Kota
}

\begin{abstract}
Random Packet CDMA, a novel packet-based multiple access scheme for connectionless, uncoordinated random channel access is proposed. Random Packet CDMA, or RPCDMA, utilizes a novel packet format which consists of a short header and a data portion. Each header is spread with a unique spreading code which is identical for all users and packets, while the data portion of each packet is spread by a randomly chosen spreading sequence. The receiver operates in two stages: header detection and data detection. For header detection a conventional spread spectrum receiver is sufficient. Headers are spread with a large enough processing gain to allow detection even in severe interference. The data portion is decoded with a sophisticated receiver, such as a multiuser detector, which allows for successful decoding of overlapping active packets. It is shown that the RP-CDMA system is detector capability limited and that it can significantly outperform Spread ALOHA systems whose performance is limited by the channel collision mechanism. RPCDMA also experiences a much smaller packet retransmission rate than conventional or Spread ALOHA, and provides better spectral efficiencies.
\end{abstract}

Index Terms-Random Packet Networks, RP-CDMA, ALOHA, Spread ALOHA, CDMA, multiuser detection.

\section{INTRODUCTION}

$\mathbf{P}$ ACKET broadcasting is a powerful strategy to share resources wherein a common channel simultaneously carries the communications of multiple uncoordinated users. In such an environment, data from users are split into packets and transmitted in an uncoordinated fashion over a common transmission channel. Headers containing address and control information are added to the packet before transmission to identify ownership and assist in initial synchronization functions.

The ALOHA system developed at the University of Hawaii ushered in the era of modern random access communication systems, whose current use includes such popular communications technologies as the Ethernet. In a random multiaccess communication environment, users transmit information whenever they have something to send, independent of each other. Information may be lost because of collisions between packets from different users. Collision resolution strategies are needed to ensure reliable transmission of data; for example,

Manuscript received December 24, 2003; revised January 28, 2005; accepted June 17, 2005. The associate editor coordinating the review of this paper and approving it for publication was M. Sawahashi.

C. Schlegel is with the Department of Electrical and Computer Engineering, University of Alberta, Edmonton, Alberta, Canada T6G 2V4 (e-mail: schlegel@ee.ualberta.ca).

R. Kempter and P. Kota are with the Department of Electrical and Computer Engineering, University of Utah, $50 \mathrm{~S}$. Central Campus Drive, Salt Lake City, UT 84112 (e-mail: kempter@eng.utah.edu).

Digital Object Identifier 10.1109/TWC.2006.03713. unsuccessful packets are queued and a retransmission is attempted after a random delay. Throughput obtained with the original ALOHA system is $18 \%$ of that of fully coordinated access to the channel. This throughput can be improved to $36 \%$ by dividing time into slots and restricting users' transmissions to these slots. Using carrier sensing methods, throughput in the order of $48 \%-58 \%$ is obtained by using complex collision resolution protocols like tree algorithms, first-come-first-serve splitting algorithms and last-come-firstserve splitting algorithms [11]. Interestingly, if channel load information is unknown, random access - in particular the ALOHA method - remains essentially the sole way of initially accessing a network by a new user [5].

Code Division Multiple Access (CDMA) [24] is a transmission method whereby each user is assigned a unique identification sequence or spreading code. Knowledge of this code is necessary for detection at the receiver. Simultaneously, interference from other active users is suppressed by the processing gain of the spreading codes. CDMA thus enables multiple users to communicate simultaneously over the entire band of the spectrum. Without sophisticated reception methods, the spectral efficiency of CDMA is quite modest, in particular if different users generate largely different received power levels due to the variable transmission environment. Nevertheless, CDMA's versatility and robustness accounts for its popularity in current and future wireless networks such as wide-band CDMA systems [20].

Our proposed random access packet system uses CDMA at the physical level, but in contrast to conventional CDMA, a common signature sequence is assigned to all packet headers. These headers are short in length compared to the data portion, and their throughput efficiency therefore does not need to be high. Separation of these headers is comfortably achieved by a Spread ALOHA receiver [1], [2]. We logically separate the entire packet traffic into header and data traffic. We show that the header traffic in our novel system is light, and that Spread ALOHA is therefore efficient. Data traffic, which makes up the bulk of the overall traffic, requires much more efficient accessing than the header traffic. All data packets are spread with unique, randomly chosen spreading sequences. They are therefore identifiable even if multiple data packets overlap, as long as a suitable detector can separate the different transmissions, and as long as the joint channel capacity is not exceeded. In spectrally tight requirements, joint detection of concurrent CDMA transmissions is proposed. Such joint detectors have the added advantage that different power levels are advantageous to detection [12] - in contrast to conventional correlation reception of CDMA. 


\section{A. Literature Survey}

Raychaudri in [30] analyzed a synchronous time slotted random access single code CDMA system. Yener and Yates in [27]-[29] investigate the specific problems of asynchronous single code random access packet switched CDMA systems. The access model in both these papers is Spread ALOHA, as proposed by Abramson in [1]. In [27], Yener and Yates propose the use of a universally known $m$-sequence to be added before the "user-identification" of the packet for detection of new active packets as well as timing recovery (see [27], Section 1, paragraphs 1 and 2). However, the system in [27] is a single-code CDMA system. There, the "user-identification" fulfills only timing recovery purposes and cannot be compared to the functionality provided by the code-ID in our proposed RP-CDMA system. In RP-CDMA, apart from timing recovery, the code-ID enables the terminals to use randomly chosen spreading sequences per packet, independent of any base station. In their original paper [27], Yener and Yates propose a two stage detector, with a multiuser receiver for stage 2 . In the follow up paper [28], assuming successful detection of new users, the authors show that having this multiuser receiver as stage 2 does not improve system throughput of their (Spread ALOHA) system because the system remains channel collision limited. This fact is due to the spreading of the entire packet by the common spreading code. In RP-CDMA, instead of spreading the overall packet with the common sequence, we propose a packet specific identifier which contains the randomly and independently chosen spreading sequence for the payload portion. Now, only the headers are collision limited (Spread ALOHA), and a multiuser detector in the base station can be used to resolve multiaccess interference and increase system capacity, see Section 9. In contrast to Yener and Yates in [27]-[29], the RP-CDMA system is not collision but only multiuser detector limited. In summary, Yener and Yates in [27]-[29] analyze an asynchronous singlecode CDMA system and show that multiuser detection cannot improve system performance. With RP-CDMA, we present a method that allows the use of random spreading sequences for a connectionless fully asynchronous CDMA system. As a result, multiuser detection can substantially increase system performance.

A basic throughput analysis of random access CDMA is given in [17], where attainable throughputs are calculated if joint detection is used. It is concluded that in a slotted system the average throughput approaches $K$, the capability of the joint detector, as $K$ becomes large. The system studied in [17] is packet-synchronized, and implicitly assumes that the receiver has knowledge of all accessing users and can detect the presence of any one of them, which makes the receiver very complex for large numbers of joint users.

In [31], an architecture for $3 \mathrm{G}$ wireless systems is described which employs ATM in the forward and Spread ALOHA in the return link. In fact, compared to the systems described in [2], [4], [27]-[30]; [31] does not provide novel ideas but presents an analysis of the combination of ATM and classical Spread ALOHA under the assumption that user traffic (packet or data traffic) is asynchronous. Under this specific assumption, the Spread Aloha return channel in [31] can be found to be lightly loaded. In contrast to [31], our proposed system supports a fully random fully asynchronous, connectionless architecture without any need for the prior exchange of user resp. code information with a base station receiver. Unlike [31], the "light" load of the access channel (virtual header channel that carries only header information) is a direct consequence of the nature of our novel packet format. By this, RP-CDMA enables high traffic on the channel (data+header traffic), thus improving the performance of random channel accessing techniques particularly under high load. Summarizing, in our proposed system, the virtual Spread ALOHA header channel is lightly loaded due to the nature of our packet. In [31], the Spread ALOHA return channel is lightly loaded only because of the asynchronous nature of the traffic models that were used.

As stated previously, if channel state information - e.g the parameter for channel access - is unknown, random channel access is the only possibility to gain entrance to the network. In 3G CDMA networks, this is facilitated by means of the RACH channel [5]. The RACH channel is a Spread ALOHA channel which is used to request payload channel resources for a specific node, e.g a specific spreading sequence. Similarly to our proposed system, it uses different spreading sequences for payload transmission. However, while in RP-CDMA, each and every packet consists of a Spread ALOHA header and a payload frame, the RACH channel is only used for initial node-base station contact. After successful reception of a RACH message, the base station broadcasts an ACK, telling the requesting node which channel resources to use - thus describing a resource reservation process with is not necessary in our novel system. We want to recall at this point that RPCDMA operates fully asynchronously; whereas as a result of the resource reservation, 3GPP compatible networks require global synchronization.

Another possibility for random channel access is described by the IEEE 802.11 MAC layer standards [6]. Similarly to the CSMA/CD protocol for wired Ethernet [7], channel sensing with collision avoidance (CSMA/CA) is performed to try to exclusively reserve the wireless channel for node transmissions. In contrast to RP-CDMA, IEEE 802.11 technology does not allow for multiuser detection and suffers from hidden and exposed terminal problems. Furthermore, as the number of users increases, the throughput of IEEE 802.11 based networks decreases dramatically while delay increases [25]. In contrast to RP-CDMA and 3GPP technology, due to the necessity for CSMA/CA, IEEE 802.11 technology cannot be used in urban or satellite environments and is restricted to $\mathrm{SOHO}^{1} /$ hotspot scenarios.

Along these lines, RP-CDMA could be very beneficial for two-way satellite communications. Due to its large propagation delays and high transponder costs, satellite communication imposes considerable demands on the channel accessing technique. Random channel access requires some form of collision resolution which is time-critical. Also, ALOHA as the most efficient random channel accessing scheme in this environment ${ }^{2}$ can at most deliver $18 \%$ of the (very expensive) channel's inherent capacity [1]. On the other

\footnotetext{
${ }^{1}$ Small Office/Home Office

${ }^{2}$ we restrict ourselves to asynchronous networks, slotted ALOHA requires synchronization
} 
hand, channel reservation schemes such as specified in the DVB-RCS standards [8], require sophisticated satellite-hub and terminal devices. Furthermore, as the network gets large, channel reservation messages for the return link can consume a considerable amount of bandwidth on the forward channel. Even worse, due to reservation request/reply communication, delays in the packet transmission process in the order of $300 \mathrm{~ms}$ and more are introduced (GEO-stationary orbit). RP-CDMA on the other hand delivers collision rates that are orders of magnitude smaller than those of (Spread) ALOHA systems which leads to less retransmissions due to packet collisions; possibly delivering very similar performance as assignment schemes. Furthermore, the only limit for packet transmission in RP-CDMA is channel capacity.

\section{ORGANIZATION}

In this paper we show that completely uncoordinated random access with a novel packet format can achieve throughputs which approach the capability of joint detection. We present and motivate a practical signalling and accessing method which requires no knowledge of the accessing users at the receiver and that works with a multiuser detector which requires receiver processing only for active packets.

The paper is organized in as follows: In Section III we describe the new packet format in detail and discuss the feasibility of the required processing components of the proposed system. In Section IV we calculate the throughput of the proposed system and show that RP-CDMA can achieve full channel capacity in the limit of large joint detection capability. Packet retransmission rates, spectral efficiencies and throughput comparisons of the proposed system compared to the benchmark protocol - Spread ALOHA - are presented in Section V. Finally, we conclude this paper with a discussion section.

\section{SYSTEM DESCRIPTION}

RP-CDMA, our proposed packet random multiple access system, supports a fully connectionless network architecture. A transmission packet of length $L_{d}$ consists of header and data frames as illustrated in Figure 1. The header frame of length $L_{h}$ consists of the access preamble and code identifier (code-ID). The access preambles of length $L_{a}$ are identical for all users using a fixed, predetermined spreading sequence. The data portion of the packet is spread by a random spreading sequence, whose identification is contained in the code identifier (code-ID). The sequence is chosen randomly and independently by the transmitter. The data portion of the packet is typically 2000-20000 bits long and hence the header constitutes only a small overhead (see Figure 4). In this paper we assume that the transmitted signals are affected by Additive White Gaussian Noise (AWGN) with a one-sided power spectral density of $N_{0}$. This basic channel model is used to present the system and study its feasibility. It is not intended as a model for a realistic wireless transmission channel. However, for such realistic wireless channels, we argue that only basic demodulation methods need to be revisited, and that typical system behavior is similar as for the AWGN channel - see concluding section.

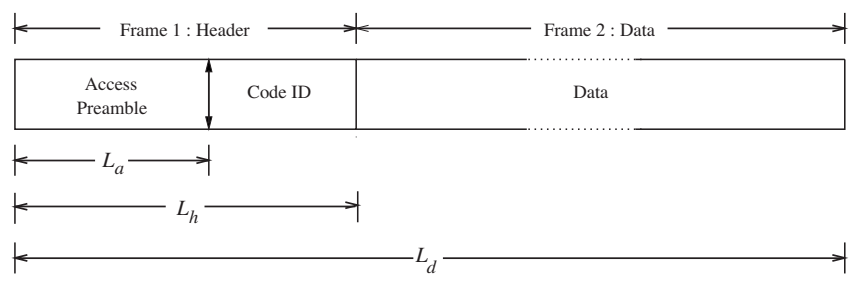

Fig. 1. Packet structure of the proposed random packet access system.

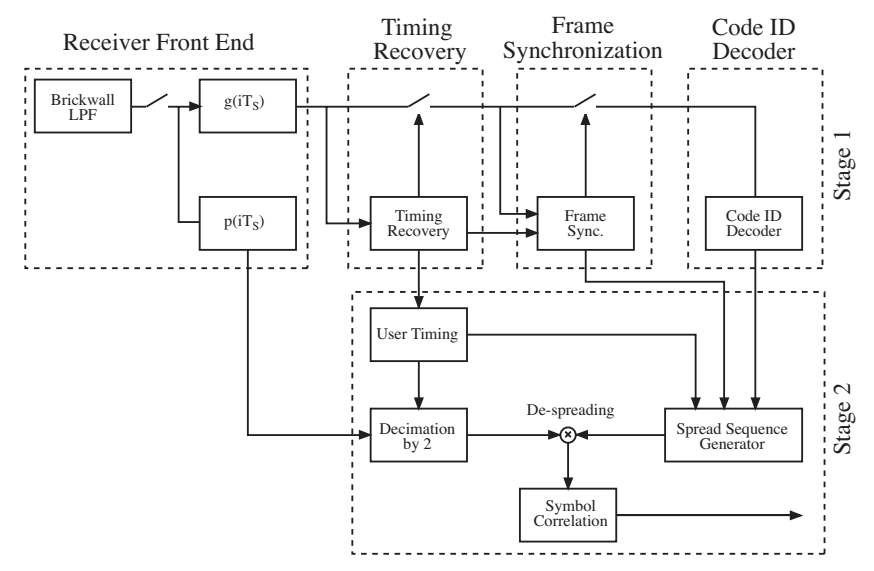

Fig. 2. Descriptive block diagram of receiver.

The wireless front-end receiver for the proposed system operates in two stages as indicated in Figure 2. The first stage deals with header detection, i.e., determination of the presence of a packet, timing recovery, and decoding of the code-ID. This information is used in the second stage of the receiver which deals with data detection.

The first stage of the receiver constantly monitors the channel for the presence of new active packets. If a new packet is detected, its timing is extracted, the code-ID is decoded, and a new packet reception process is spawned off at stage II which is dedicated to the decoding of this packet. The stage II process performs packet specific despreading and matched filtering and passes this data to the decoder, which may employ sophisticated joint detection methods to jointly decode concurrent packets.

\section{A. Stage I}

1) Basic Operation: Stage I of the receiver deals with header detection and basic parameter extraction. Its front-end is an asynchronous filter matched to the chip pulse waveform, sampled at some adequate sampling rate. It next performs timing recovery according to an asynchronous digital method [9], which allows the receiver clock to be asynchronous to the transmitter clocks - an essential requirement for uncoordinated multiple access. Once timing is found, the code-ID is decoded which contains packet information such as the particular random spreading sequence used in the data portion of the packet. An $m$-sequence can, for example, be used for this purpose. This synchronization information is supplied to the code-ID decoder and the spreading sequence generator which generates a symbol-synchronized, packet-specific spreading sequence for demodulation. Even for moderate lengths of 
the data spreading sequence $N$, the probability that the data portions of two simultaneously active packets have the same spreading sequence is very small. Typically, a random number generator of length $N$ will deliver $2^{N}-1$ different sequences.

Exact timing information, frame synchronization, and codeID information are passed on to Stage II, which may be a software process dedicated to the detection of the packet. Stage I now becomes free again to search for new packet arrivals. It is tantamount for our system that Stage I is capable to detect new packets even in heavy interference, i.e., even if many packet transmissions are already in progress. This is ensured in the following way: since the packet header forms only a small fraction of the entire packet, it may be spread with a larger processing gain than the payload data. The active packets are spread by random sequences which constitute random interference that can be suppressed by the header processing gain to manageable levels, allowing header detection even in the presence of many active packets. Note that in the data detection stage increasing the processing gain is not possible without loosing bandwidth, which is why a multiuser detector is necessary for Stage II for high spectral efficiency.

2) Timing recovery: Timing acquisition in our random access scenario is synonymous with packet detection, since if the acquisition circuit detects a valid timing point, an active header transmission has been detected. Since there may be multiple concurrent transmissions in progress, timing acquisition in high signal-to-interference ratios is fundamental for successful operation of the proposed system. For this reason, in the following we discuss timing recovery limitations in some more detail. Clearly, an in-depth discussion is beyond the scope of this paper, but our low-SNR timing recovery algorithm has been thoroughly tested and patented [9].

The signal at the receiver front end during a received packet is

$$
\begin{aligned}
y(t) & =\sum_{j=1}^{J} x_{j}\left(t-\tau_{j}\right)+n(t) \\
& =x_{J}\left(t-\tau_{J}\right)+n(t)+I(t) \quad t \in\left[0, L_{d} T_{b}\right]
\end{aligned}
$$

where $J$ is the number of active packets, $L_{d}$ is the length of the packets in data symbols, $\tau_{j}$ is the delay in arrival of the $j^{\text {th }}$ packet and $T_{b}$ is the symbol duration. It is assumed that $0 \leq \tau_{j} \leq L_{d} T_{b}$. The $j^{t h}$ transmitted packet is expressed as

$$
\begin{aligned}
x_{j}(t) & =\sum_{i=0}^{L_{a}-1} a_{h}(i) s_{h}\left(t-i T_{h}\right) \\
& +\sum_{i=L_{a}}^{L_{h}-1} a_{c}\left(i-L_{a}\right) s_{h}\left(t-i T_{h}\right) \\
& +\sum_{i=L_{h}}^{L_{d}-1} a_{d}\left(i-L_{h}\right) s_{j}\left(t-i T_{b}\right)
\end{aligned}
$$

where $a_{h}(i)$ is the $i^{t h}$ symbol of the common access preamble and $s_{h}(t)$ is the access preamble spreading sequence of duration $T_{h}$ and is expressed as

$$
s_{h}(t)=\sum_{m=0}^{G-1} \beta_{m} g\left(t-m T_{c}\right)
$$

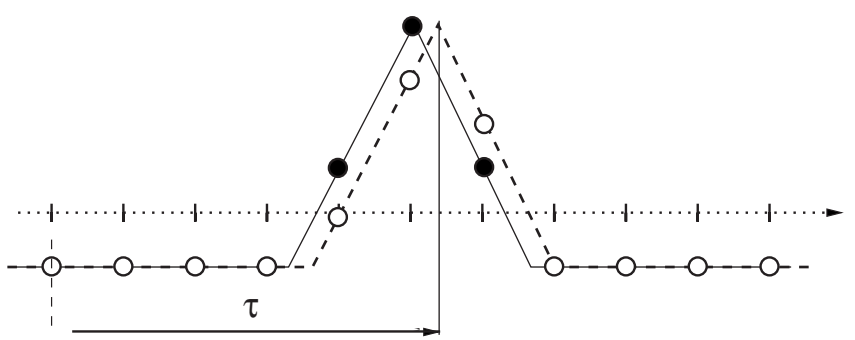

Fig. 3. Signal output and sample point example from a spread spectrum matched filter

where $G$ is the header processing gain, i.e., the number of chips per symbol (whereas $N$ is the data processing gain), $T_{c}$ is the chip duration, $\beta_{m} \in\{-1 / \sqrt{N}, 1 / \sqrt{N}\}$ is the $m^{t h}$ spreading chip of the access preamble and $g(t)$ is the unitenergy chip waveform. $G$ is chosen as an integer multiple of $N$ to facilitate the derivation of the data timing from the acquired header symbol timing. We are making our considerations with rectangular chip waveforms $g(t)$ for simplicity, but more bandwidth efficient pulse waveforms are used in actual stateof-the-art implementations [24]. The symbol $a_{c}(i)$ is the $i^{t h}$ element of the block encoded code-ID block. $a_{d}(i)$ is the $i^{t h}$ bit of the data block and $s_{j}(t)$ is a random spreading sequence used in the data portion of the $j^{\text {th }}$ user. It has duration $T_{b}=(N / G) T_{h}$ and is expressed as

$$
s_{j}(t)=\sum_{m=0}^{N-1} \alpha_{m, j} g\left(t-m T_{c}\right)
$$

where $N$ is the processing gain of the data spreading sequence and $\alpha_{m, j} \in\{-1 / \sqrt{N}, 1 / \sqrt{N}\}$ is the $m^{t h}$ spreading chip of user $j$.

The timing detector is concerned only with the access preamble, but must operate in strong interference $I(t)$. With this in mind, the matched filter receiver for the preamble is given by

$$
\mathrm{g}_{\mathrm{MF}}\left(T_{h}-t\right)=\sum_{m=0}^{G-1} \beta_{m} g\left(T_{h}+m T_{c}-t\right)
$$

The transmitter and receiver operate with asynchronous, independent timing clocks to allow completely uncoordinated access. However, clock accuracy on the order of $1 \mathrm{ppm}$ is feasible [13]. This means that the sampling clocks will drift no more than 1 sampling interval once every 1 million samples, and since typical packet sizes are about two orders of magnitude smaller, the transmitter/receiver timing clock frequency mismatch can safely be ignored.

Using a suitable spreading sequence such as an $m$-sequence, the symbol-periodic outputs of both the in-phase and quadrature channels of the matched filter, $z(t)$ and $z\left(k T_{s}\right)$ (in the absence of noise), look approximately as sketched in Figure 3 , where the dots indicate the discrete samples taken by using two samples/chip. A maximum likelihood estimate of the delay is calculated as [19],

$$
\hat{\tau}=\arg \max _{\tau} E\left(\left|z\left(n T_{b}+\tau\right)\right|^{2}\right)
$$


From $\hat{\tau}$, we can calculate an integer sampling point $m_{n}$, and a fractional offset $\mu_{n}$, which accounts for the asynchronicity between transmitter and receiver clocks, i.e., the offset between the solid and open dots in Figure 3. These values are given as

$$
m_{n}=L_{\text {int }}(\hat{\tau}), \quad \mu_{n}=\hat{\tau}-m_{n} T_{s}
$$

The sampled taps of the symbol matched filter in the presence of noise and interference are given by

$$
z_{k}=z\left(k T_{s}\right)= \begin{cases}s\left(k T_{s}\right)+n_{k} ; & \text { taps containing signal } \\ n_{k} ; & \text { taps with no signal }\end{cases}
$$

where $n_{k}$ is complex Gaussian noise and interference with variance $I_{0} / 2$ in each dimension and $s\left(k T_{s}\right)$ is the signal at tap $k$, and is time and tap dependent as evident from Figure 3.

The digital timing recovery circuit [9] operates a timevarying interpolation filter which shifts the sample points such that they are symmetrically centered, shown by the solid dots in Figure 3. A dynamic analysis of this process is beyond the scope of this paper and we assume that the taps are aligned with the transmitter clock for a simplified feasibility analysis.

Since $z\left(k T_{s}\right)$ is Gaussian, $\left|z_{k}\right|^{2}$ is $\chi^{2}$ distributed [21]. Due to the triangular autocorrelation, which is a result of the spreading sequence, and with centered timing, i.e., $\tau=k T_{s}$, one of the squared taps will have signal value $E_{s}$, the adjacent taps have signal values $E_{s} / 4$ and all other taps have signal value energy $E_{s} / G^{2} \approx 0$ (see Figure 3 ), where $E_{s}$ is the symbol energy.

In a real system, the timing procedure needs to accumulate tap values to bring the signal level sufficiently above the noise floor to ensure accurate sample point detection. Peak detection of accumulated tap values is performed at the timing detector, noting that $\left(s^{2}\left(p T_{s}\right)\right)$ is periodic with $N_{t s}$. Due to the lack of phase coherence at this point, for tap $p$, the algorithm calculates the accumulated tap energies

$$
w_{p}=\sum_{j=0}^{L_{a}}\left|z_{k-j N_{t s}}\right|^{2}
$$

where $N_{\mathrm{ts}}=L_{\mathrm{int}}\left(\frac{T_{b}}{T_{s}}\right)$, and $p=k \bmod N_{\mathrm{ts}}$. These tap values are $\chi$-square distributed [21] with $2 L_{a}$ degrees of freedom, and $E\left[\omega_{p}\right]=L_{a}\left(E_{s}+I_{0}\right)(G / N)$ for the center tap, and $E\left[\omega_{p}\right]=L_{a}(G / N) I_{0}$ for the noise taps.

The sample point detection problem is now that of accurately identifying timing mismatch $\tau$. Assuming for simplicity that we have the synchronized samples, i.e., $\tau=k T_{s}$, the tap $w_{\tau}$ should be the maximum value, i.e., $w_{\tau}>w_{p}, p \neq \tau$. Working with a frame of $M=2 G$ sample values and a detect threshold $d_{\mathrm{th}}$, the probability of correct detection is given by

$$
\begin{aligned}
P(\text { detect })= & P\left(\omega_{\tau}>d_{\mathrm{th}}\right) \prod_{\substack{p=1 \\
(p \neq \tau, \tau-1, \tau+1)}}^{M} P\left(\omega_{p}<d_{\mathrm{th}}\right) \\
& \prod_{i=-1,1} P\left(\omega_{\tau+i}<d_{\mathrm{th}}\right)
\end{aligned}
$$

On the other hand, the probability that one of the noise samples is mistakenly identified as a valid timing point, or one of the samples adjacent to the maximum sample, is given by

$$
P(\text { false })=1-P\left(w_{\tau+1}<d_{\mathrm{th}}\right)^{2} P\left(w_{\kappa}<d_{\mathrm{th}}\right)^{M-3}
$$

Note that this is also an upper bound on the probability that a header detection is signaled when there is no packet header transmission in progress.

These probabilities can be calculated in a straightforward way by noting that all the $w_{p}$ are $\chi$-square distributed, with cumulative probability density functions

$$
F_{W}^{(c)}(w)=1-e^{-w / I_{0}} \sum_{k=0}^{2 L_{a}-1} \frac{1}{k !}\left(\frac{w}{I_{0}}\right)^{k} \quad \text { noise taps }
$$

$F_{W}^{(n)}(w)=1-Q_{L_{a}}\left(\sqrt{\frac{L_{a} E_{s} G / N}{I_{0} / 2}}, \sqrt{\frac{w}{I_{0} / 2}}\right)$ signal taps

where $Q_{m}(w / \sigma, \sqrt{w} / \sigma)$ is the generalized Marcum's $Q$ function [21].

The length of the access preamble depends on the number of symbols needed to obtain a high probability of correctly identifying the timing point with a low probability of false alarms. Figure 4 shows the probability $\left[F_{W}^{(c)}(w)\right]^{M}$ that all noise taps are smaller than $w$, as well as the probability $F_{W}^{(n)}(w)$ that the center tap is smaller than $w$, for $G E_{s} /\left(N I_{0}\right)=3 \mathrm{~dB}$, a header length of $L_{a}=50$, and $M=64$. The value of $3 \mathrm{~dB}$ corresponds to a CDMA system where the number of active packets is equal to half the (header) processing gain $G \geq N$ of the system. For example, if $G=2 N$, this means that $N$ concurrent packets can be active, which is a very high system load. As can be seen, a threshold of $d_{\text {th }}=w=80$ will produce header detection error rate $\approx 0$, since all noise taps are smaller than $w=80$, and the probability that the center tap is smaller $w=80$ is less than $10^{-5}$. This is meant to demonstrate that accurate header detection is feasible even in a high interference environment with a header size no more than about $1 \%$ of a typical packet size. Of course, in a practical system the detection thresholds as well as the header length need to be determined carefully given all the system parameters. Additionally, different received power levels, as are to be expected in random access systems, will adversely affect this calculation, requiring either longer headers, or more header spreading, both of which are synonymous.

3) Stage II: Once timing is acquired, a packet's presence has been detected successfully. We have argued in the previous section, that this process can be performed reliably. The second stage of the receiver has to extract the payload data from the data frames of these packets. Depending on the bandwidth efficiency required, this detector may need to employ sophisticated joint detection methods. Such joint detection methods - for example interference cancellation, decorrelation, projection receivers or more sophisticated turbo iterative decoding strategies [3], [18], [22], [23], [26] - can all be potentially used in the second stage of the receiver. However, for ease of a flexible implementation, we envision the use of iterative decoders where additional users can easily be added or dropped corresponding to the varying numbers of jointly active packets. 


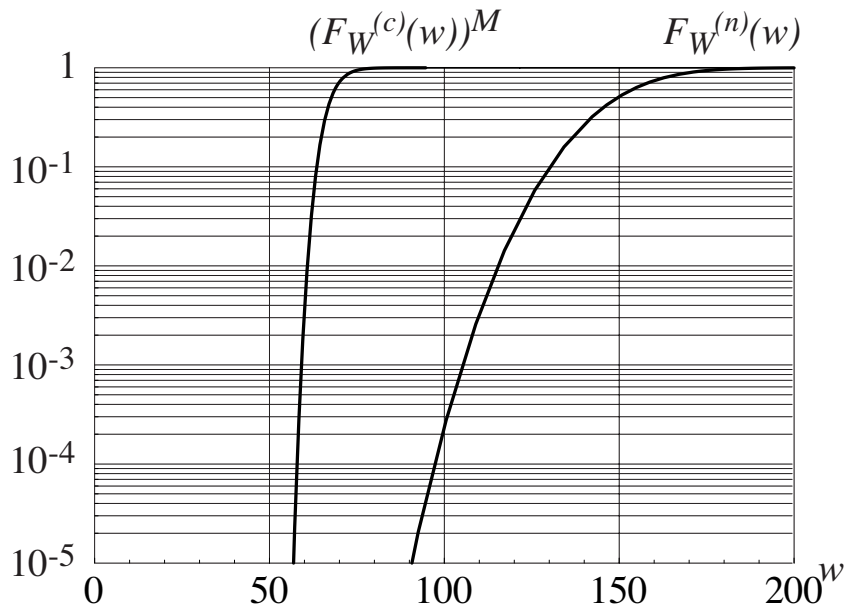

Fig. 4. Cumulative probability density of the center tap and $M$ noise taps, for $M=64, L_{a}=50$, and $G E_{s} /\left(N I_{0}\right)=3 \mathrm{~dB}$, illustrating that $d_{\mathrm{th}}$ would provide reliable header detection.

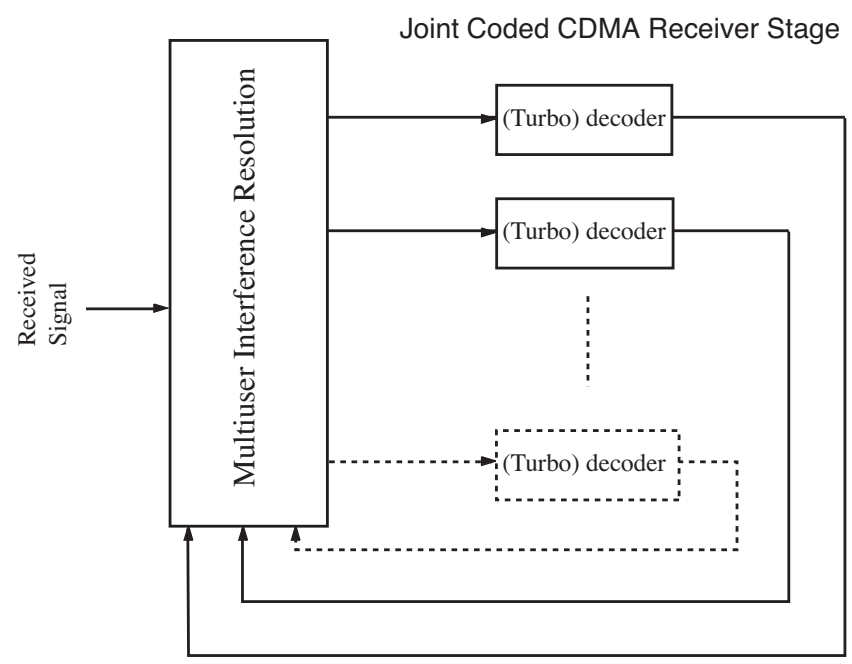

Soft Information “'recycled"

Fig. 5. Basic diagram of a "layered" multiuser detector of the type discussed in [3], [12], [23].

The basic structure of such iterative multiuser detectors [3], [23] is shown in Figure 5, and consists of a simple interference suppression stage and a bank of parallel single channel decoders. The interference suppression stage is the only part of the receiver which needs to be laid out for the maximum number of users. In the systems studied in [3], [12], [23] this stage is a simple interference cancellation process where interfering signals are subtracted from the received signal. The single user decoders can be software processes which can be spawned off as required. Such iterative receivers have been studied extensively, and the performance results of the receivers presented in [23] will be used later in this paper to obtain spectral efficiency figures. It is furthermore known that such joint receivers work very well in environments with different received power levels. In fact, from the joint detector point of view, the equal received power situation presents a worst-case situation.

\section{System ThroughPUT}

In this section, we calculate the system throughput, defined as the total number of successful accesses to the system. All calculations are normalized to the header length $L_{h}$, which is constant for all users, and, as we will see, is an important system parameter.

Such system throughputs have been calculated for a packetsynchronized slotted system as envisioned in future wide-band CDMA applications by Liu et. al. [17], who found that in the limit of large joint detection capability, denoted by $K$, the system throughput asymptotically approaches $K$ under idealized assumptions.

In our system, initial packet transmission failure can occur in two ways: failure in detecting the header or failure in joint detection. Hence, successful transmission is defined as successful header detection and successful joint detection. Let $P_{p}$ denote the probability of packet survival, $P_{h}$ denote the probability of successful header detection and $P_{m}$ denote the probability of successful joint detection. Hence,

$$
P_{p}=P_{h} P_{m}
$$

Header access in RP-CDMA is identical to Spread ALOHA. However, with regards to our specific worst case receiver which can process only one header at a time, we now make the pessimistic assumption that each packet header needs a traffic-free interval of $2 L_{h}$ for its successful transmission. Due to the spreading, even overlapping headers can potentially be detected according to the Spread ALOHA mechanism [1], reducing the vulnerability time. We will see, however, that header collisions do not represent a biting system limitation, which is why simplified processing can be assumed. In fact, the "header channel", i.e., all the header traffic, is very light compared to the overall traffic due to the large ratios $L_{d} / L_{h}$, and hence a simple ALOHA mechanism is efficient.

Assuming a Poisson arrival of packets ${ }^{3}$ at rate $\lambda$ packets/header length,

$$
P_{h}=e^{-2 \lambda}
$$

For successful joint detection, the total interfering traffic should be less than the capability of the joint detector. Assuming a detector capable of detecting $K$ users, we require that there are no more than $K$ active packets in a time span $2 L_{d}$ of two packets, and

$$
P_{m}=P\left(J \leq K-1 \mid \text { during } 2 L_{d}\right)
$$

According to the Poisson process,

$$
P(J=k)=e^{-2 \lambda \frac{L_{d}}{L_{h}}} \frac{\left(2 \lambda \frac{L_{d}}{L_{h}}\right)^{k}}{k !}
$$

Using (IV) and (5), we obtain

$$
P_{m}=\sum_{k=0}^{K-1} e^{-2 \lambda \frac{L_{d}}{L_{h}}} \frac{\left(2 \lambda \frac{L_{d}}{L_{h}}\right)^{k}}{k !}=f\left(K, 2 \lambda \frac{L_{d}}{L_{h}}\right)
$$

\footnotetext{
${ }^{3}$ More realistic traffic scenarios as well as achievable capacities are presented in [16], where a network perspective of this system is taken.
} 
The function $f(K, \nu)$ converges to the step function $S\left(\frac{\nu}{K}\right)$ as $K \rightarrow \infty$ [17], where

$$
S(x)=\left\{\begin{array}{cc}
1 & \text { if } x<1 \\
0.5 & \text { if } x=1 \\
0 & \text { if } x>1
\end{array}\right.
$$

This shows that in the limit the multiuser detector always provides successful detection as long as the arrival rate $\lambda<$ $\left(L_{h} / L_{d}\right) /(K / 2)$, i.e., the average arrival rate per packet is not larger than $K / 2$. This is a consequence of the law of large numbers, and it is important to note that this holds in the limit. Comparisons with realistic values of $K$ are shown in Figures 6 and 7 below, which are somewhat smaller than $K / 2$. Note that for a slotted system the capacity can be twice as large [17], just as with Classical ALOHA.

The Poisson distribution in (6) asymptotically converges to a Gaussian distribution [15] with mean and variance $2 \lambda \frac{L_{d}}{L_{h}}$. Hence,

$$
\begin{aligned}
P_{m} \stackrel{\stackrel{L_{d}}{L_{h} \rightarrow \infty} \longrightarrow}{\longrightarrow} \int_{0}^{K-1} \frac{1}{\sqrt{4 \pi \lambda \frac{L_{d}}{L_{h}}}} \exp \left(-\frac{\left(x-2 \lambda \frac{L_{d}}{L_{h}}\right)^{2}}{4 \lambda \frac{L_{d}}{L_{h}}}\right) d x \\
\quad \approx \quad 1-\mathrm{Q}\left(\frac{K-1-2 \lambda \frac{L_{d}}{L_{h}}}{\sqrt{2 \lambda \frac{L_{d}}{L_{h}}}}\right)
\end{aligned}
$$

Using (4), the probability of packet success $P_{p}$ can now be represented as

$$
P_{p}=e^{-2 \lambda}\left(1-\mathrm{Q}\left(\frac{K-1-2 \lambda \frac{L_{d}}{L_{h}}}{\sqrt{2 \lambda \frac{L_{d}}{L_{h}}}}\right)\right)
$$

and the system throughput in [packets/packet duration] becomes

$$
R=\lambda \frac{L_{d}}{L_{h}} e^{-2 \lambda}\left(1-\mathrm{Q}\left(\frac{K-1-2 \lambda \frac{L_{d}}{L_{h}}}{\sqrt{2 \lambda \frac{L_{d}}{L_{h}}}}\right)\right)
$$

In Figure 6, this system throughput, which is normalized to the packet size $L_{d}$, is plotted against the arrival rate $2 \lambda \frac{L_{d}}{L_{h}}$ in packets/packet duration for the case of a joint detector with capability $K=10$ (see (6)). Figure 6 also illustrates the effect of packet size on system throughput and the limitations on system throughput imposed by the multiuser detector and header collisions. Note that for $\frac{L_{d}}{L_{h}}>100$, throughput is limited by the multiuser detector capability according to (9) and header collisions become negligible.

Figure 7 shows the same plot with a multiuser detection capability of $K=50$. It becomes evident that the limiting throughput $\rightarrow K / 2$ quite slowly, reaching $72 \%$ for $K=50$, that is, the large detector capability limit calculated in [17] is quite challenging to approach with realistic values of the joint detection capability.

\section{Channel acCess via CDMA}

We now return to the question of physical channel access, which in RP-CDMA is accomplished via CDMA as discussed in Section III. This is the same accessing method

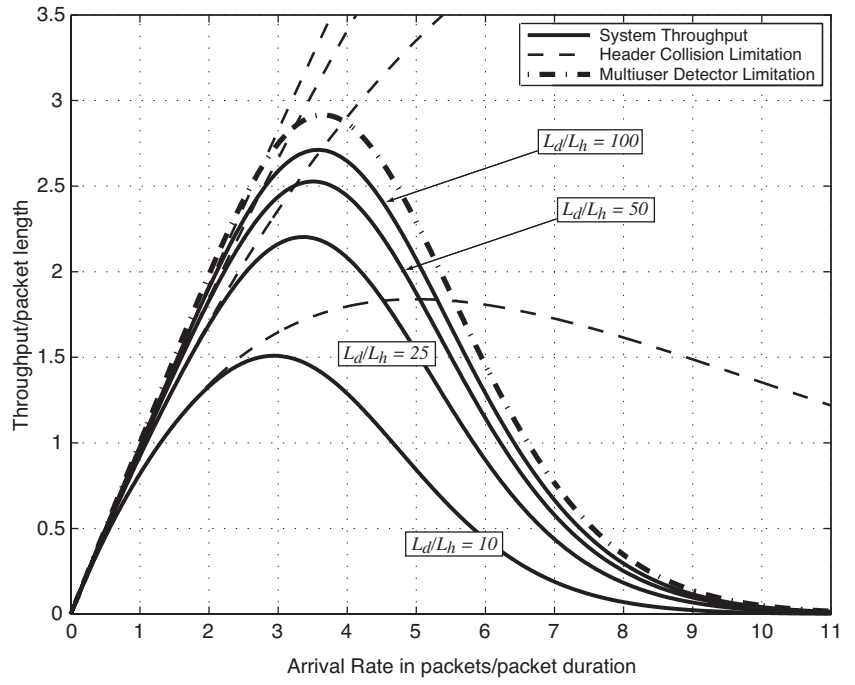

Fig. 6. Throughput for varying packet sizes for a multiuser detection capability of $K=10$.

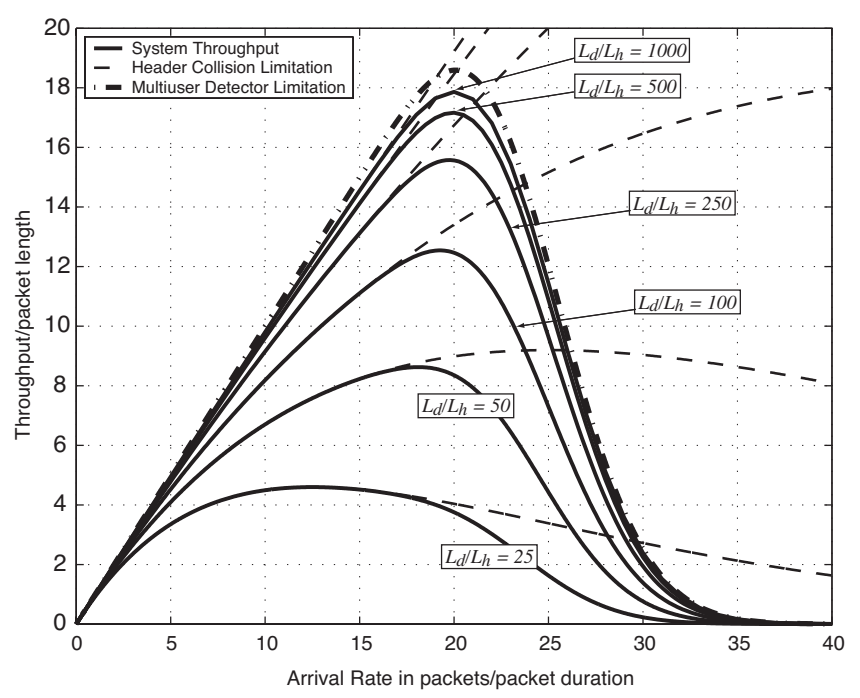

Fig. 7. Throughput for varying packet sizes for a multiuser detection capability of $K=50$.

used in Spread ALOHA [1], which we use as a benchmark for packet retransmission rates, i.e., the probability of transmission failure at any particular attempt, throughput and spectral efficiency. Spread ALOHA uses a bandwidth greater than the signal bandwidth by the spreading factor $N$. There, signals are modified by spreading each bit in time, with all users employing the same spreading sequence. The ordinary ALOHA contention protocol is used to control access. At the receiver, the asynchronous timing of the packets means that unless packets collide within a chip time, concurrent transmissions are suppressed by the processing gain and can be successfully decoded. Nonetheless, it turns out that the throughput of Spread ALOHA is identical to the Classical ALOHA system [1]. Our new proposed system differs from Spread ALOHA in its use of random spreading sequences, which means that the new system is detector performance limited whereas Spread ALOHA is collision limited. 


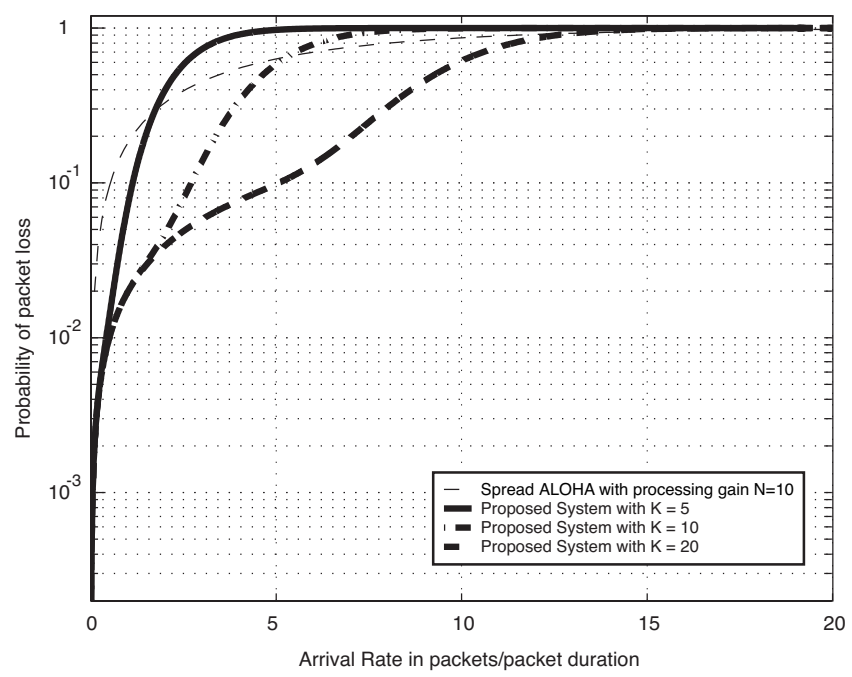

Fig. 8. Probability of packet retransmission

Spread ALOHA can in fact be seen in terms of the concepts developed here in the following way. Spread ALOHA is equivalent to having a collision vulnerable zone, $L_{h}$, which is the total packet length $L_{d}$ divided by the processing gain $N, L_{h}=L_{d} / N$. Furthermore, Spread ALOHA does, strictly speaking, perform concurrent (joint) detection, where the interfering packets, suppressed by the processing gain of the spread spectrum system, are treated as noise. As seen later, this fixed packet length to header ratio $L_{d} / L_{h}$, and the limited capability of the receiver is what limits the performance of Spread ALOHA compared with the proposed RP-CDMA system.

Differences also result in the retransmission performance. Figure 8 shows the probability of packet retransmission for various detector capabilities of the proposed system and Spread ALOHA. For the proposed system operating at the optimal traffic rate for Spread ALOHA and with $K=20$, the probability of packet retransmission is $10 \%$ as compared to $60 \%$ in Spread ALOHA. This reduced packet retransmission rate results in smaller buffer sizes in the proposed system, offsetting some of the complexity of the receiver.

In Figure 9, the throughput of the proposed system and Spread ALOHA are plotted against the arrival rate $\lambda L_{d} / L_{h}$ in packets/packet duration. Depending on how much interference Spread ALOHA can tolerate, the throughput changes according to formulas analogous to those derived in Section IV. Values for three cases of detector capability for the proposed RP-CDMA system are plotted: $K=N / 2, N$, and $K=2 N$, and for two values of the interference tolerance for Spread $\operatorname{ALOHA}(N / 5$ and $N)$. Clearly, RP-CDMA can achieve significantly higher throughput given good joint detector capability, with maximum throughput values close to the arrival rate.

Figure 9 also compares spectral efficiencies of the proposed system with Spread ALOHA, shown by the axis on the right hand side. In order to achieve the high level of joint detection considered, we assume that an iterative multiuser detector [23] using a rate $R=1 / 3$ error control code is employed, resulting in the required $E_{b} / N_{0}$ values indicated in the figure. For comparison we also assumed that Spread ALOHA

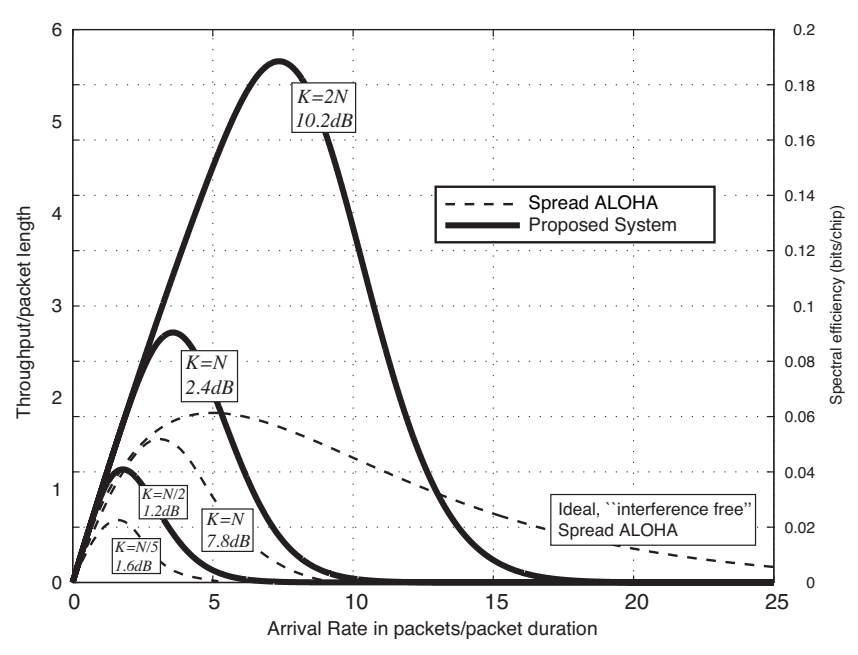

Fig. 9. Throughput comparison with Spread ALOHA

employs a turbo code of approximately equal complexity. Since Spread ALOHA does not employ joint detection, a required $\left[E_{b} / I_{0}\right]_{\text {req }}=0.8 \mathrm{~dB}$ for the turbo code considered [10] translates to an actual $E_{b} / N_{0}=1.55 \mathrm{~dB}, E_{b} / N_{0}=3.0 \mathrm{~dB}$ for $K=N / 2, E_{b} / N_{0}=7.8 \mathrm{~dB}$ for $K=N$, and values larger than $K=1.247 N$ cannot be supported anymore since the required $\left[E_{b} / I_{0}\right]_{\text {req }}$ for the turbo code is no longer attainable.

The signal-to-noise ratio $E_{b} / N_{0}$ is calculated from the required signal-to-interference ratio as follows:

$$
\begin{aligned}
{\left[\frac{E_{b}}{I_{0}}\right]_{\text {req. }} } & =\frac{E_{b}}{2(\text { noise variance }+ \text { multiuser interference })} \\
& =\frac{E_{b}}{2\left(\frac{N_{0}}{2}+\frac{K}{N} E_{s}\right)}
\end{aligned}
$$

Hence, from (10), the actual $E_{b} / N_{0}$ needed is

$$
\frac{E_{b}}{N_{0}}=\frac{\left[\frac{E_{b}}{I_{0}}\right]_{r e q .}}{1-\frac{2 R K}{N}\left[\frac{E_{b}}{I_{0}}\right]_{r e q .}}
$$

In the light of this discourse and Figure 9 it becomes evident how Spread ALOHA is collision limited, whereas RPCDMA can increase its throughput as more resources become available in the form of signal-to-noise ratio, and/or detector capability.

\section{CONCLUSION}

RP-CDMA, a novel packet-based, fully asynchronous random multiple access method is proposed, analyzed, and compared to Spread ALOHA. RP-CDMA introduces a novel packet structure which uses a common signature sequence for the packet header and random spreading for the data portion. By this, joint detection methods can be used in the base station receiver which leads to higher throughput, higher spectral efficiencies, lower retransmission rates and smaller buffer sizes compared to Spread ALOHA. In fact, a comparison of the spectral efficiencies of RP-CDMA with Spread ALOHA assuming an iterative turbo decoding scheme shows, that the spectral efficiency of RP-CDMA can be several times higher than that of Spread ALOHA. It is shown that 
our novel system can achieve throughputs which approach the Shannon limit of the channel as the joint detector capability becomes large. Network studies using various traffic models [16] have shown that RP-CDMA can achieve in the order of $70 \%-90 \%$ of the Shannon capacity of the multiple access channel. This is due to the fact that RP-CDMA is only detector capability limited, rather than collision limited as any other random channel accessing technique. Since all the information required for packet decoding is contained in the packet itself, the system enjoys a high degree of versatility. Furthermore, it possesses inherent channel access fairness due to the lightly loaded "header channel".

\section{ACKNOWLEDGEMENTS}

The authors wish to acknowledge Dr. Zhenning Shi for providing the results on joint detection used in this paper.

\section{REFERENCES}

[1] N. Abramson, "Fundamentals of packet multiple access for satellite networks," IEEE J. Select. Areas Commun., vol. 10, no. 2, pp. 309316, Feb. 1992.

[2] N. Abramson, "VSAT data networks," Proc. of the IEEE, vol. 78, pp. 1267-1274, July 1990.

[3] P. Alexander, A. Grant, and M. Reed, "Iterative detection in codedivision multiple-access with error control coding," European Trans. Telecommun., vol. 9, no. 5, pp. 419-426, Sept.-Oct. 1998.

[4] N. Abramson, "Fundamentals of packet multiple access for satellite networks," IEEE J. Select. Areas Commun., vol. 10, no. 2, pp. 309-316, Feb. 1992

[5] The Random Access Channel, RACH, 3GPP Technical Specifications $25.211,25.213,25.214$

[6] The IEEE802.11 MAC-Layer specification, http : //grouper.ieee.org/groups/802/11/

[7] The IEEE802.3 CSMA/CD (ETHERNET) specification, http : //www.ieee802.org/3/

[8] The DVB-RCS standard, ETSI EN 301 790, http : //www.etsi.org

[9] Z. Bagley and C. Schlegel, "Implementation of digital timing recovery," L-3 Communications patent application filed with Perman \& Green, LLP, Fairfield, CN.

[10] S. Benedetto and G. Montorsi, "Serial concatenation of interleaved codes: performance analysis, design, and iterative decoding," IEEE Trans. Inform. Theory, vol. 44, no. 3, pp. 909-926, May 1998.

[11] D. Bertsekas and R. Gallager, Data Networks. Englewood Cliffs, NJ: Prentice-Hall, 1992.

[12] J. Boutros and G. Caire, "Iterative multiuser joint decoding: unified framework and asymptotic analysis," IEEE Trans. Inform. Theory, vol. 48, no. 7, pp. 1772-1793, July 2002.

[13] Chase Specific Company's CG50. http : //www.chase2000.com/cg50/CG50_991227.pdf

[14] B. P. Crow, I. Widjaja, L. G. Kim, and P. T. Sakai, "IEEE 802.11 wireless local area networks," IEEE Commun. Mag., vol. 35, pp. 116-126, Sept. 1997.

[15] W. J. Feller, An Introduction to Probability Theory and its Applications. John Wiley and Sons, vol. 2, 1971.

[16] R. Kempter and C. Schlegel, "Capacity and QoS analysis for a novel packet based wireless access system," in Proc. Vehicular Technology Conference (VTC) 2003, invited paper.

[17] Q. Liu, E.-H. Yang, and Z. Zhang, "Throughput analysis of CDMA systems using multiuser receivers," IEEE Trans. Commun., vol. 49, no. 7, pp. 1192-2102, July 2001.

[18] R. Lupas and S. Verdu, "Linear multiuser detectors for synchronous code-division multiple-access channels," IEEE Trans. Inform. Theory, vol. 35, pp. 123-136, Jan. 1989.

[19] H. Meyr, M. Moeneclaey, and S. A. Fechtel, Synchronization, Channel Estimation and Signal Processing, Volume 2, Digital Communication Receivers. John Wiley and Sons, vol. 2, Oct. 1997.

[20] L. B. Milstein, "Wideband code division multiple access," IEEE $J$. Select. Areas Commun., vol. 18, pp. 1344-1354, Aug. 2000.

[21] J. G. Proakis, Digital Communications, McGraw Hill College Div., 1995.
[22] C. Schlegel, S. Roy, P. Alexander, and Z. Xiang, "Multi-user projection receivers," IEEE J. Select. Areas Commun., vol. 14, Oct. 1996.

[23] Z. Shi and C. Schlegel, "Joint decoding of serially concatenated coded CDMA," IEEE J. Select. Areas Commun., Special Issue: Multiuser Detection Techniques, vol. 19, no. 8, Aug. 2001.

[24] A. J. Viterbi, CDMA: Principles of Spread Spectrum Communications. Addison-Wesley, Reading, 1995.

[25] T. Ho and K. Chen, "Performance evaluation and enhancement of the CSMA/CA MAC protocol for 802.11 wireless LAN's," in Proc. PIMRC 1996, pp. 392-396.

[26] Z. Xie, R. Short, and C. K. Rushforth, "A family of suboptimum detectors for coherent multiuser communications," IEEE J. Select. Areas Commun., vol. 8, pp. 683-190, May 1990.

[27] A. Yener and R. D. Yates, "Multiuser access detection for CDMA systems," in Proc. CISS'98, pp. 17-22.

[28] A. Yener and R. D. Yates, "Multiuser access capacity of packet switched CDMA systems," in Proc. 49th IEEE Vehicular Technology Conference 1999, vol. 2.

[29] A. Yener and R. D. Yates, "Acquisition dependent random access for connectionless CDMA systems," in Proc. IEEE WCNC 2000, vol. 1, pp. 173-178.

[30] D. Raychaudri, "Performance analysis of random access packetswitched Code Division Multiple Access systems," J. Select. Areas Commun., vol. 29, pp. 895-901, June 1981

[31] N. Guo, S. D. Morgera, and P. Mermelstein, "Common Packet Data Channel (CPDC) for integrated wireless DS-CDMA networks," J. Select. Areas Commun., vol. 14, pp. 735-749, May 1996.

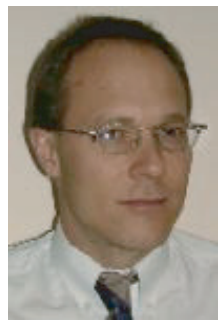

Christian Schlegel received the Dipl. El. Ing. ETH degree from the Federal Institute of Tec hnology, Zurich, in 1984, and the M.S. and Ph.D. degrees in electrical engi neering from the University of Notre Dame, Notre Dame, IN, in 1986 and 1989

He held academic positions at the University of South Australia, the University of Texas and the University of Utah, Salt Lake City. In 2001 he was named iCORE Professor for High-Capacity Digital Communications at the University of Alberta, Canada. His interests are in the area of error control coding and applications, multiple access communications, digital communications, as well as analog and digital implementations. He is the author of the research monographs "Trellis Coding" and "Trellis and Turbo Coding" by IEEE Press, as well as "Coordinated Multiple User Communications," co-authored with Professor Alex Grant, published by Springer. Dr. Schlegel received an 1997 Career Award, and a Canada Research Chair in 2001.

Dr. Schlegel is an associate editor for coding theory and techniques for the IEEE Transactions on Communications, and a guest editor of the IEEE Proceedings on Turbo Coding. He served as the technical program co-chair of ITW 2001 and ISIT'05. He was also the general chair of the CTW'05, as well as on numerous technical program committees.

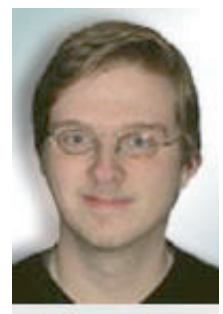

Roland Kempter received the Dipl. Ing (FH) degree in Applied Physics from the University of Applied Sciences, Weingarten, Germany in 2000 From 2000-2002, he was with NDSatCom, Europe's largest supplier of satellite communication equipment. He was involved in the development of BBI, the world's first DVB-RCS compliant two-way satellite communication system. Also, he developed signaling and scheduling mechanisms for the West Early Bird system study, the first satellite with onboard packet switching capabilities sponsored by

ESA. He received the M. Eng degree in Electrical and Computer Engineering from the University of Utah, USA in 2005 and is currently working towards his Ph.D. degree.

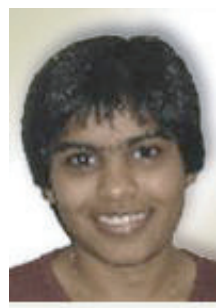

Preeti Kota received her engineering degree in India, and her MSEE degree from the University of Utah in 2001 with a thesis on random access techniques. 